\title{
Nouvelle Sociologie Économique et Nouvelle Géographie Économique Leur impact en analyse territoriale
}

\section{Christophe Mager, Lausanne et Neuchâtel}

\section{Introduction}

L'accentuation de la globalisation de l'économie mondiale et la persistance des déséquilibres économiques, dans un contexte généralisé de reprise de la croissance, ont multiplié les interrogations sur les meilleures façons d'apprécier globalement la performance économique d'un territoire et de simuler ses possibilités d'évolution sous des conditions définies, pour luimême et par rapport à d'autres espaces de référence.

Même si l'on pourrait croire que les façons de répondre à ces questionnements se standardisent, et simplifient d'autant la tâche de l'analyste, la complexité reste de mise. «Faire science» en analyse territoriale oblige ainsi le plus souvent à naviguer entre différentes théories, aucune n'étant susceptible d'expliquer la totalité des enjeux rencontrés. La littérature montre que l'analyse des disparités économiques régionales et l'identification des modalités régionales d'accumulation de capital physique tend à passer par la mobilisation d'auteurs se rattachant à au moins deux représentations de l'économie et des sociétés, l'une relevant de la Nouvelle Géographie Économique, l'autre de la Nouvelle Sociologie Économique. Leur cooccurrence dans le champ de la recherche territoriale ne se fait toutefois pas avec le même poids, la Nouvelle Géographie Économique étant plus largement sollicitée.

Bien que les postulats de la Nouvelle Géographie Économique et de la Nouvelle Sociologie Économique s'opposent, leur complémentarité analytique respective ne fait aucun doute. Mettant en question la légitimité de la partition disciplinaire entre économie et sociologie, il est posé que l'évaluation territoriale géographique ne devrait pas choisir entre l'une et l'autre de ces ressources, mais les utiliser conjointement.

\section{La Nouvelle Géographie Économique: le primat du marché}

La Nouvelle Géographie Économique se bâtit sur l'idée que seul le marché permet de révéler à la communauté humaine la juste et vraie valeur des biens qu'elle produit, et que, à ce titre, les marchés sont les moins imparfaits des mécanismes de coordination (Foucault 2004; Postel 2008). Leur libre fonctionne- ment engendre des avantages pour tous, sans qu'aucun accord coercitif ne soit nécessaire, pour autant que les acteurs agissent indépendamment de toute réflexion éthique ou politique, mus par leur seule rationalité économique (la maximisation de leur intérêt) et leurs préférences subjectives. La Nouvelle Géographie Économique de KRUGMAN (1995) s'inscrit dans cette perspective.

Trois éléments constituent le modèle de base de la Nouvelle Géographie Économique (Krugman 1991): un secteur géographiquement non mobile à rendements d'échelle constants, produisant, en concurrence parfaite, des biens homogènes; un secteur mobile produisant, avec des rendements d'échelle croissants, un ensemble de marchandises diversifiées dans un contexte de concurrence monopolistique. La résolution d'un tel modèle pour un système à régions multiples ou à espace continu génère des paysages économiques complexes très proches de celui du système de places centrales de Lösch (1954).

À la différence de celui-ci, cette solution s'insère parfaitement dans le cadre de la théorie de l'équilibre général et est obtenue grâce à l'intégration de deux hypothèses inspirées du modèle de Dixit \& STiglitz (1977). Toutes les variations de production dans le secteur monopolistique sont des variations du nombre de firmes qui le composent, la production par entreprise restant constante. Les consommateurs souhaitent une offre de produits diversifiée, ce qui fait que l'importance du marché est un indicateur d'attractivité, au contraire des modèles antérieurs, où la taille du marché n'était pas pertinente.

Dans un tel cas - même si les producteurs et les consommateurs sont initialement répartis de manière quasi-homogène et même si tous les endroits de l'espace sont identiques en termes de dotation en ressources et en technologie -, deux forces déséquilibrantes constituant des propriétés purement émergentes du modèle agiront (KRUGMAN 1996). Les premières sont de type backward: les entreprises qui se déplacent vers certains lieux y accroîtront la demande, et, de ce fait, les rendront encore plus attractifs. Les deuxièmes forces sont de type forward: plus de production diversifiée dans les zones où les entreprises se concentreront permettra aux consommateurs d'y acheter la même gamme de produits à un prix plus avantageux, en raison de coûts de transport inférieurs. Dans le cas où les facteurs immobiles ou les biens non échangea- 
bles sont essentiels à la production (comme le travail) et à la consommation (comme le logement), une force centrifuge apparaîtra, encourageant à la dispersion des activités (Fujita, Krugman \& Venables 1999).

La simplicité et la plasticité du modèle de Krugman en a fait la fécondité. FuJita \& THISSE (2003) montrent qu'il est possible de rattacher ce modèle à la théorie de la croissance endogène, en y insérant deux paramètres nouveaux, soit le capital humain et l'acquisition de brevets. Ils mettent en évidence que ce sont les secteurs d'activité modernes et innovants qui se regroupent vers les centres lorsque les coûts de transport diminuent et que, pour autant que la croissance économique soit rapide, un pattern centre-périphérie génère - paradoxalement - plus d'avantages économiques pour une région en périphérie que si elle était située dans un espace homogène, sans centre bien circonscris.

Le modèle de KRUGMan permet aussi d'explorer l'effet des conflits politiques sur la taille des villes, en fonction de la croissance économique et de qui contrôle la majorité dans un vote (Henderson \& BeCKER 2000), ou encore de comprendre pourquoi la diversité des villes en augmente la productivité (Duranton \& Puga 2001).

La Nouvelle Géographie Économique apparâ̂t ainsi comme capable d'expliquer l'essentiel des faits stylisés relevant de l'analyse territoriale (p.ex. concentration, exurbanisation, décentralisation, délocalisation, spécialisation), en rendant superflue toute considération d'ordre historique ou culturel. D'une grande cohérence, capable de jeter des ponts entre les théories de la localisation et celles de la croissance, la Nouvelle Géographie Économique occupe une place dominante.

La Nouvelle Sociologie Économique de Granovetter (1985) conteste la légitimité de cette position, considérant que le concept sur lequel est bâtie la Nouvelle Géographie Économique est illusoire, soit celui d'un marché autonome permettant l'accord des individus en dehors de toute perspective collective et de tout autre mode de lien social.

Partant, selon Granovetter, si la Nouvelle Géographie Économique est un référentiel théorique et un outil analytique puissant, en refusant de s'interroger sur les conditions de l'émergence et du fonctionnement des marchés et en ne prenant en compte que les externalités pécuniaires (positives ou négatives) agissant sur les interactions locales entre les offres et/ou les demandes marchandes, la Nouvelle Géographie Économique ne pourrait légitimement prétendre expliquer, à elle seule, l'hétérogénéité socio-spatiale. La Nouvelle Sociologie Économique entre en jeu.

\section{La Nouvelle Sociologie Économique: l'importance des institutions}

La Nouvelle Sociologie Économique, telle que définie par GranovetTER, s'est essentiellement construite autour du refus de la relégation de la sociologie économique dans une périphérie analytique complexée. Faisant de l'irrespect des postulats des approches néoclassiques une condition sine qua non à une interprétation pertinente des modalités de la lutte que les hommes mènent contre la rareté, la sociologie économique de Granovetter refusait de n'être, à la PARsons (1956), que la science des causes et conséquences socio-spatiales des choix économiquement irrationnels (SWEDBERG 1994).

Doutant de la systématique pertinence de l'individualisme méthodologique, faisant de la rationalité maximisatrice non plus un postulat mais une possibilité et considérant que les relations sociales préexistent toujours à l'économie - et, partant, au marché - GranoVETTER choisissait de ne plus seulement côtoyer, mais affronter l'orthodoxie économique, y compris dans ses extensions beckeriennes. En cela, la Nouvelle Sociologie Économique allait s'opposer, dès le début des années 1990, à la Nouvelle Géographie Économique de Krugman.

Reste que caractériser cette sociologie de nouvelle peut étonner, compte tenu du fait que l'on retrouvait déjà chez Durkheim (1908), Simiand (1912) et Veblen (1898) l'idée selon laquelle l'économie néoclassique n'était pas acceptable en l'état:

«Leur conception de la sociologie économique les entraine à chercher un nouveau fondement général alternatif à l'ancienne économie politique» (GISLAIN \& STEINER 1995: 67). Simiand écrivait:

«Telle que nous la concevons, la science économique positive s'oppose, d'une part, et tout autant, à l'économie politique traditionnelle, dite abstraite ou pure, que, d'autre part, à l'historicisme économique ou à la description pure et simple des faits» (Gislain \& Steiner 1995: 73).

Granovetter (2004: 121), tout se démarquant des approches sur-socialisées de la sociologie classique et des approches sous-socialisées de l'économie, énonce trois postulats:

«Ma critique se fonde sur trois assertions, dont chacune dérive de la tradition sociologique classique: 1) la poursuite d'objectifs économiques s'accompagne normalement de celle d'autres objectifs de nature non économique (...);2) l'action économique (comme toute action) est socialement située et encastrée dans le réseau des relations personnelles $(. .) ; 3$.$) les institutions économi-$ ques (comme toutes les institutions) sont socialement construites». 
Cette Nouvelle Sociologie Économique peut être ramenée à deux propositions:

- Pour fonctionner, le marché doit, en univers incertain, être enchâssé dans des relations sociales ou morales créatrices de liens suffisamment forts pour que l'on puisse s'assurer, lors des échanges, que chaque contractant va assurer sa part de responsabilité. De ce fait, les acteurs économiques ne peuvent se considérer comme affranchis de toute référence extérieure. Celle-ci peut être une convention, un sens commun, un modèle cognitif, un système de connaissance ou encore un «principe d'action justifiable», construisant les informations jugées nécessaires à l'action et déterminant la nature des objets qui peuvent servir de ressources (EYMARD-DuvemaY et al. 2006). Partant, la prise en compte de ce référentiel est le passage obligé qui permet de comprendre pourquoi un marché fondamentalement non autorégulateur et myope peut pourtant conduire à des périodes plus ou moins prolongées de croissance stable.

- Chaque individu exerce ses choix en étant influencé par les institutions qui forment la société dans laquelle il vit. En retour, ces choix individuels contribuent à faire évoluer les institutions, sans déterminisme dans un sens ou dans l'autre. Il n'y a pas une forme institutionnelle universelle qui serait le marché, mais plusieurs grandes catégories d'institutions dont on peut faire l'histoire et la géographie - en repérant la variété des institutions dans une économie donnée ou entre économies nationales. Ainsi, la Nouvelle Sociologie Économique considère qu'il n'existe pas une réalité institutionnelle a-temporelle et a-spatiale (Clerc 2008).

La rupture avec la sociologie à la PARSONs est consommée. Cette dernière,

«parce qu'elle se concentrait sur les pré-conditions institutionnelles de la vie économique, n'avait pas besoin de proposer une description alternative de l'activité économique quotidienne» (GRANOvetTER 2000a: 203).

La Nouvelle Sociologie Économique

«renverse l'impérialisme économique et propose des analyses sociologiques de thèmes économiques fondamentaux, tels que les marchés, les contrats, l'argent, le commerce et la banque» (GranovetTer 2000a: 203).

L'analyse de l'espace social de la finance, dont celui des bourses d'échange (figure 1), prend une importance croissante (HeIlbron 2003).

La Nouvelle Sociologie Économique ne saurait être réduite à la Nouvelle Économie Institutionnelle de COASE (1972). Même si elle partage avec la Nouvelle Sociologie Economique certaines hypothèses sur le comportement des agents (par exemple leur rationalité limitée ou leur propension aux comportements opportunistes), la Nouvelle Économie Institutionnelle réfléchit moins à la construction sociale des économies, comme le fait GranOVETTER, qu'à celle des dispositifs de régulation alternatifs au marché mis en place par les agents pour minimiser les coûts de transaction, dont les entreprises et les réseaux (NoRTH 1990; WILLIAMSON 1985).

Appliquée à l'analyse géographique, la Nouvelle Sociologie Économique fait du territoire un niveau de cohérence sociétale spatialement inscrit où le vécu s'accroche aux contraintes formelles et aux représentations collectives, sorte de mélange d'ordre naturel et d'ordre construit intégrant le fond de connaissances diffuses, de comportements et de disciplines intériorisés dans la vie sociale (BLOCK 2002).

L'espace densifié institutionnellement est, dans cette optique, un «producteur d'accords» économiques, capable de fixer les principes moraux nécessaires pour assurer l'efficacité de la gestion des ressources et diminuer les coûts de transaction engendrés par la crainte de voir un partenaire de l'échange tirer parti d'une information asymétrique. Un tel espace engendre une offre de spécificité territoriale - ou de gouvernance de nature à «ancrer» des entreprises, voire à en attirer d'autres (GRANOVETTER 2000b).

À chaque territoire correspondra alors une dynamique économique particulière. Les régions se trouvent impliquées dans un processus d'irréversibilité dynamique, où chaque nouvel équilibre dépend du parcours effectué depuis la situation initiale. Ce qui fait que la variété des trajectoires institutionnelles qu'une région peut emprunter n'est pas définie, mais est contenue dans un «cône des trajectoires possibles» (MARCELPOIL 1997). Ce n'est guère qu'entre les régions partageant les mêmes arrangements institutionnels qu'il pourra y avoir convergence (MACLEOD \& GoodwIN 1999).

Si la performance et l'attractivité régionales sont envisagées comme étroitement liées à la densité et aux caractéristiques des relations qui s'y jouent (CAPELLO \& NiJKAMP 1995), ces dernières sont très rétives à la mesure et rendent l'usage de méthodes qualitatives indispensables. KRUgman les qualifie d'ailleurs de notions floues:

«Son argument est qu'en matière spatiale les coûts d'accès physique, qui augmentent ou diminuent localement la désidérabilité des biens, constituent, après tout, la seule donnée concrète, les autres effets étant par nature très difficiles à lier à un cadre spatial donné» (Veltz 1996: 73).

En ce sens, la Nouvelle Géographie Économique et la Nouvelle Sociologie Économique explorent des aspects complémentaires de l'économie capitaliste (BOYER 2007): la première se focalise sur les compor- 


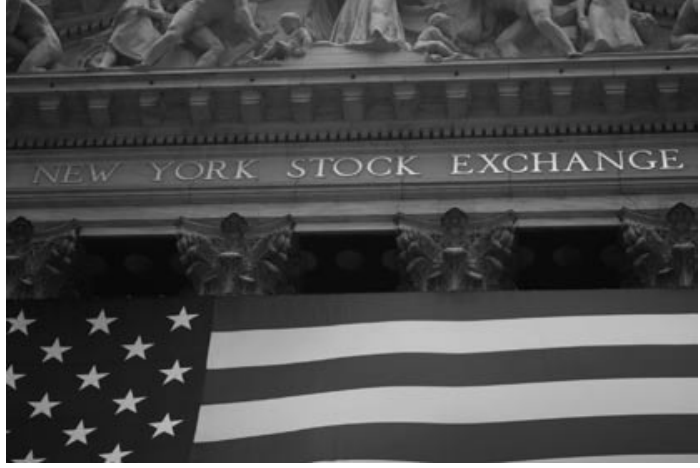

Fig. 1: La bourse de New York: un construit social The New York Stock Exchange: a social construct Die New Yorker Börse: ein soziales Konstrukt Photo: M. Roque DE PINHO

tements déterminés par la seule rationalité instrumentale maximisatrice; la seconde explore l'autonomie, les comportements stratégiques et la capacité d'innovation des acteurs, les institutions et les organisations générées, de même que leurs interactions. Ainsi,

«à rebours du mouvement qui porte les sciences sociales

à diviser le travail intellectuel, à spécialiser les recher-

ches, (...), il est nécessaire et utile de faire se rencontrer les théories économiques et sociologiques de manière à fournir de meilleures explications des faits économiques que ne le font l'un et l'autre de ces deux savoirs lorsqu'on les met en œuvre d'une manière isolée ou, pire, contradictoire» (STEINer 1999: 3).

De ce fait, la Nouvelle Géographie Économique et la Nouvelle Sociologie Économique devraient être des référents familiers aux géographes. Un tel bilinguisme les dotant des connaissances transversales indispensables pour créer, d'une part, les conditions spatiales favorables à l'ancrage et l'exercice des activités économiques, et, d'autre part, pour assurer la pérennité des processus d'innovation compétitive (PECK 2005). On en est loin, la Nouvelle Sociologie Économique n'inspirant encore qu'à la marge la géographie économique.

\section{Une géographie économique encore très largement néoclassique}

La Nouvelle Sociologie Économique a favorisé, en géographie économique, tant l'analyse de l'espace socialement réseauté - celui des entreprises principalement - que celle des contextes macro-institutionnels à l'origine de la diversité des capitalismes. Le premier axe de recherche est à bien des égards relaps (PECK 2005), souvent myope, comme dans la Nouvelle Éco- nomie Institutionnelle, quant au contexte territorial, privilégiant les analyses topologiques et mécanistes des relations migratoires ou strictement marchandes (Rozenblat \& Tissandier 2007). La seconde orientation, notamment sous l'influence des démarches régulationnistes ou culturalistes (BARNES 2001), a doté la géographie économique d'un nouvel arsenal fécond, lui permettant d'expliciter hors des sentiers rebattus la spatialité historicisée des économies.

Dans cette perspective, afin d'incarner dans leurs analyses l'idée selon laquelle ce sont les institutions et leurs enchaînements historiques qui sont à l'origine des régularités économiques et de leurs mutations constatées dans une période et un espace donnés, les analystes territoriaux s'inspirant de la Nouvelle Sociologie Économique tendent à recourir à divers outils conceptuels dont celui de forme institutionnelle, définie comme la codification à des fins de socialisation d'un ou de plusieurs des rapports sociaux qu'entretiennent les différents agents économiques, dont l'État, et qui sont au cœur du passage d'une régularité partielle à la régulation d'ensemble, composant un mode de régulation. Ce sont ces institutions-clés qui permettent aux agents la projection de leurs subjectivités et de leurs intérêts, suivant des modalités qui restent compatibles avec une relative stabilité des processus d'accumulation du capital physique dans un espace donné (régime d'accumulation). Parmi les différentes formes institutionnelles composant un mode de régulation, la géographie économique d'inspiration sociologique analyse de manière privilégiée, en les inscrivant spatialement (CREVOISIER 2007):

- la politique économique: comprenant les composantes du régime monétaire (p.ex. création monétaire, systèmes financiers) et budgétaire (p.ex. dépenses sociales, subventions), la législation relative aux entreprises et à la protection sociale;

- la relation d'emploi: qui désigne non seulement le mode de construction sociale du salariat en tant que sujet politique collectif et agent économique inséré dans des relations d'échange, mais aussi une codification juridico-contractuelle, puisqu'elle est à la fois: le système de rémunération; la construction des qualifications et l'orientation des mobilités éducatives et professionnelles (rapport éducatif ou rapport professionnel); la construction des relations de pouvoir (rapport organisationnel); les formes de régulation des conflits (rapport conflictuel);

- la concurrence: les relations entre les producteurs;

- l'adhésion au régime supra-régional/international: c'est-à-dire les modalités de l'articulation des niveaux géographiques d'un même espace national ou 
celles de l'insertion de l'économie nationale/locale dans l'économie mondiale (p.ex. analyse du degré d'ouverture aux échanges de biens, de la dépendance capitalistique);

- le territoire: soit le triple recouvrement d'une proximité organisationnelle, liée aux interpénétrations des processus productifs; d'une proximité institutionnelle, liée aux résolutions coordonnées des problèmes productifs par des logiques d'action collective fondées sur des institutions locales qui peuvent être soit formelles (collectivités territoriales, organismes professionnels), soit informelles (normes tacitement adoptées par les acteurs locaux); et d'une proximité spatiale, favorisant la diffusion des connaissances et du savoir-faire entre les protagonistes du territoire, et étroitement liée aux effets de milieu en contribuant à la création de systèmes de valeurs et de représentations collectives.

La Nouvelle Sociologie Économique a ainsi incité la géographie économique à aller au-delà de l'étape habituelle de l'identification des systèmes de production et spatiaux, en recourant à une approche mésoanalytique visant à appréhender des formes productives intermédiaires marquées localement par une dynamique à la fois commune (individus ayant des buts communs et se coordonnant grâce au résultat même de leurs interactions) et globale (permettant la coordination «à distance» d'organisations distinctes) (Pecqueur 1996, 2000).

La géographie économique s'est ainsi attelée à expliciter territorialement le degré de stabilité des schémas coopératifs d'interaction, l'importance du principe de réciprocité, la volonté d'ouverture sur l'extérieur, les réseaux de relations hors marché qui existent entre les entrepreneurs et la circulation de connaissances informelles ou formelles que ces réseaux permettent (AMIN \& Thrift 1994; Becattini 1992; Markusen 2000; Peet 1997; SAXENIAN 1994; WALLISER 2000).

Reste qu'il ne s'agit là que d'une géographie économique, somme toute marginale en termes de représentation académique. Comme le soulignait BECATTINI (1992), le mainstream, soit la Nouvelle Géographie Économique, dans laquelle la fiction de l'homme économique et le «déracinement» dominent, ne fait guère de place aux approches pour lesquelles l'encastrement socioculturel est un élément analytique à ne surtout pas négliger. Comment comprendre cette périphéricité?

L'explication réside au moins en partie dans le fait que la géographie économique d'inspiration sociologique répond surtout à une demande sociale particulière, celle de trouver des solutions à des problèmes locaux de politique publique (p.ex. ségrégations socio-spatia- les, ajustements structurels générateurs d'inégalités spatiales, chômage). Le mode de résolution de ces problèmes, guidé par la réalité du terrain socioculturel et l'induction, s'éloigne significativement des démarches canoniques empirico-formelles de la géographie économique orthodoxe, orientée vers la création de modèles mathématiques permettant des prédictions suivies de tests empiriques.

Partant, les études dont on dispose, et qui s'inscrivent dans une perspective sociologisante, témoignent systématiquement de l'idiosyncrasie des espaces géographiques et montrent la nécessité d'expliciter les creux et les reliefs institutionnels des territoires (DAVIET 2005; Galland et al. 1993; Perkmann 2006). Paradoxalement, cette capacité à révéler la polyphonie territoriale expose la géographie économique d'inspiration sociologique à une critique récurrente: elle serait dominée par l'empirisme, l'ad hoc, le ponctuel, l'explication ex post et le monographisme, au contraire de la Nouvelle Géographie Économique, nomothétique et totalisante (BOYER 2007). De fait, la géographie économique d'inspiration sociologique pâtit des mêmes reproches faits, en son temps, à l'analyse régionale de VIDAL DE La BLACHE (1903). L'on peut comprendre que, dans un paysage scientifique faisant souvent, sous l'influence de l'essai de Friedman (1953), de l'irréalisme des hypothèses, de l'abstraction et de la simplification du monde des vertus analytiques essentielles, la géographie économique d'inspiration sociologique, ainsi accusée de particularisme, peine à s'imposer.

Face à une économie investissant, à la BECKER (BECKer \& Murphy 2001), les domaines les plus variés, comme les interactions sociales, les réseaux ou la famille, il est à craindre que la géographie économique continue de suivre la

«pente de plus forte déclinaison (et reste dans l'orbite de l'économie néoclassique), considérant que la sociologie, discipline ancillaire, (est une stricte) pourvoyeuse d'intuitions et/ou de constats empiriques que seule l'économie est à même de formaliser dans un cadre théorique cohérent» (GAUTIÉ 2004: 20-21).

\section{Bilan et perspective}

Même si les bénéfices de la spécialisation sont incontestables, il devrait être d'usage de penser, avec Morin (1997), que les ruptures de frontières et la circulation des concepts permettent de résoudre des problèmes dont la solution aurait été invisible si l'esprit hyper-disciplinaire, avec ce qu'il implique d'excluant, avait dominé.

L'analyse géographique est propice à cette inscription des réflexions à la croisée de différents champs du savoir, sans la prétention d'aboutir à un dépassement 
des disciplines, mais avec la volonté de montrer que peut s'instaurer, entre elles, un mode de coopération effectif.

Les évolutions et les amendements successifs apportés à la géographie par la Nouvelle Sociologie Économique ont ainsi permis d'établir que les performances économiques régionales dépendent de la capacité passée, présente et future des acteurs locaux à stimuler, à contrôler et à internaliser la connaissance et l'innovation, mais aussi à générer de la confiance, ou une gouvernance. Comprendre p.ex. les phénomènes de (non-)convergence des niveaux de revenu, créer localement des ressources capables de retenir et d'attirer les investissements, assurer la sécurité et la viabilité du capital investi nécessite en conséquence d'aller, en géographie économique, au-delà - sans y renoncer - de l'analyse des dotations spatiales largement étudiées par la Nouvelle Géographie Économique, soit: (1) la structure productive, c'est-à-dire l'origine et l'étendue de la spécialisation dans certaines branches, l'ampleur de l'intégration fonctionnelle des activités économiques, la viabilité des services financiers régionaux, la taille et la qualité du bassin de main-d'œuvre régional; (2) la structure spatiale, soit la situation géographique et les infrastructures (degré d'urbanisation, systèmes de transports et de communications, qualité de l'environnement).

De ce fait, l'attention portée au contexte social souhaité par la Nouvelle Sociologie Économique s'ajoute mais ne se substitue pas à l'analyse des facteurs de concurrence spatiale établis par la Nouvelle Géographie Économique.

Reste que l'analyse de la littérature par Реск (2005) montre que la géographie économique n'accorde encore qu'un strapontin à la Nouvelle Sociologie Économique, et qu'elle pourrait faire sienne la remarque de Swedberg (2006: 77):

«Il est clair que, pour la plupart des spécialistes, la science sociale la plus importante de notre temps est l'économie, plus précisément le type d'économie qui est habituellement qualifiée de mainstream et qui trouve son bastion le plus puissant aux Etats-Unis, dans le corps professionnel de ses économistes».

Pour peu que l'on considère que les faits économiques spatialisés sont des faits sociaux et que, en conséquence, ils partagent un même «modèle d'intelligibilité» (OrLÉAN 2007), on ne peut que plaider pour une géographie osant davantage traverser les frontières entre économie et sociologie.

\section{Bibliographie}

Amin, A. \& N. Thrift (éds) (1994): Globalization, institutions, and regional development in Europe.- Oxford: Oxford University Press.
BARNES, T.J. (2001): Retheorizing Economic Geography. From the quantitative revolution to the cultural turn. - In: Annals of the Association of American Geographers 91: 546-565.

Becattini, G. (1992): Le district marshallien: une notion socio-économique. - In: BenKo, G. \& A. LipIETZ (éds): Les régions qui gagnent. - Paris: Presses Universitaires de France: 35-55.

Becker, G. \& K.M. Murphy (2001): Social Economics: market behavior in a social environment. - Harvard: Harvard University Press.

BLOCK, F. (2002): Rethinking capitalism. - In: BIGGART, N.W. (éd.): Readings in economic sociology. - Oxford: Blackwell: 219-230.

BOYER, R. (2007): Capitalism strikes back. Why and what consequences for social sciences? - http://regulation.revues.org/document2142.html 15.06.2007.

CAPello, R. \& P. NijKamp (1995): Le rôle des externalités de réseau dans les performances des firmes et des régions: l'exemple des NTI. - In: RALLET, A. \& A. TORRE (éds): Économie industrielle et économie spatiale. - Paris: Economica: 273-293.

Clere, D. (2008): Le besoin d'institutions. - In: Alternatives Economiques, Hors-Série 77: 17-19.

COASE, R.H. (1972): Industrial organization: a proposal for research. - In: Fuchs, V. (éd.): Policy issues and research opportunities in industrial organization. - Cambridge: National Bureau of Economic Research: 59-73.

Crevoisier, O. (2007): Economie régionale, économie territoriale: la dynamique des milieux innovateurs. - In: Mollart, A., Sauboua, E. \& M. Hirczak (éds): Territoire et enjeux du développement régional. - Versailles: Quae: 61-83.

DAVIET, S. (2005): Industrie, culture, territoire. - Paris: L'Harmattan.

Dixit, A. \& J. STIGLITZ (1977): Monopolistic competition and optimum product diversity. - In: American Economic Review 67: 297-308.

Duranton, G. \& D. Puga (2001): Nursery cities. Urban diversity, process innovation, and the life cycle of products. - In: American Economic Review 91: 14541477.

Durkheim, E. (1908): Débat sur l'économie politique et les sciences sociales. - In: Bulletin de la société d'économie politique: 64-73.

Eymard-Duvernay, F., Favereau, O., Orléan, A., SALAIS, R. \& L. ThÉVENot (2006): Valeurs, coordination et rationalité: trois thèmes mis en relation par l'économie des conventions. - In: Eymard-Duvernay, F. (éd.): L'économie des conventions, méthodes et résultats. - Paris: La Découverte: 23-44.

Foucault, M. (2004): Naissance de la biopolitique. - Paris: Gallimard-Seuil.

Friedman, M. (1953): Essays on Positive Economics. - Chicago: University of Chicago Press.

FujITA, M. \& J.F. ThIsse (2003): Does geographical 
agglomeration foster economic growth? And who gains and loses from it? - In: Japanese Economic Review 54: 121-145.

Fujita, M., Krugman, P. \& A. Venables (1999): The Spatial Economy. Cities, regions and international trade. - Cambridge: MIT Press.

Galland, B., Leresche, J.-P., Dentan, A. \& M. BasSAND (1993): Identités urbaines. Une comparaison entre Genève et Lausanne. - Lausanne: Fonds national suisse de la recherche scientifique, Programme national de recherche $\mathrm{n}^{\circ} 30$ «Ville et transport».

Gautié, J. (2004): Les développements récents de l'économie face à la sociologie: fécondation mutuelle ou nouvel impérialisme? - Communication au premier Congrès de l'Association Française de Sociologie, Paris, février 2004; http://www.melissa.ens-cachan. fr/IMG/pdf/gautie.pdf.

Gislain, J.-J. \& P. Steiner (1995): La Sociologie économique, 1890-1920. - Paris: Presses Universitaires de France.

GranovetTer, M. (1985): Economic action and social structure: the problem of embeddedness. - In: American Journal of Sociology 91: 481-510.

Granovetter, M. (2000a): L'ancienne et la nouvelle sociologie économique: histoire et programme. - In: Granovetter, M. (éd.): Le marché autrement. Essais de Mark Granovetter. - Paris: Desclée de Brouwer: 193-222.

Granovetter, M. (2000b): Action économique et structure sociale: le problème de l'encastrement. - In: Granovetter, M. (éd.): Le marché autrement. Essais de Mark Granovetter. - Paris: Desclée de Brouwer: 75-114.

Granovetter, M. (2004): Les institutions économiques comme constructions sociales. - In: OrLÉAN, A. (éd.): Analyse économique des conventions. - Paris: Presses Universitaires de France: 119-134.

Heilbron, J. (2003): Les structures sociales de la finance. - In: Actes de la recherche en sciences sociales 146-147: 3-7.

Henderson, V. \& R. Becker (2000): Political economy of city sizes and formation. - In: Journal of Urban Economics 48: 453-484.

KRUGMAN, P. (1991): Increasing returns and economic geography. - In: Journal of Political Economy 99: 483499.

Krugman, P. (1995): Development, geography and economic theory. - Cambridge: The MIT Press.

Krugman, P. (1996): The self-organizing economy. - Cambridge: Blackwell.

Lösch, A. (1954): The economics of location. - New Haven: Yale University Press.

MacLoed, G. \& M. Goodwin (1999): Space, scale and state strategy: rethinking urban and regional governance. - In: Progress in Human Geography 23: 503-527. MarCelPoIL, E. (1997): L'organisation du Sillon Alpin: contribution à l'analyse des territoires. - Grenoble:
Institut de Recherche Economique Production Développement - Université Pierre Mendès France.

MARKUSEN, A. (2000): Sticky places in slippery space: a typology of industrial districts. - In: BENKo, G. \& A. LiPIETZ (éds): La richesse des régions. La nouvelle géographie socio-économique. - Paris: Presses Universitaires de France: 85-119.

Morin, E. (1997): Sur la transdisciplinarité. - In: La revue du Mouvement Anti-Utilitariste en Sciences Sociales (M.A.U.S.S.) 10:21-29.

OrLÉAN, A. (2007): Les ambiguïtés de la sociologie économique. - In: L'Economie politique 35: 66-70.

NoRTH, D. (1990): Institutions, institutional change, and economic performance. - Cambridge: Cambridge University Press.

Parsons, T. (1956): Economy and society. - New York: The Free Press.

PeCK, J. (2005): Economic sociologies in space. - In: Economic Geography 81: 129-175.

Pecqueur, B. (éd.) (1996): Dynamiques territoriales et mutations économiques. - Paris: L'Harmattan.

Pecqueur, B. (2000): Le développement local. - Paris: Syros.

PEET, R. (1997): The cultural production of economic forms. - In: LeE, R. \& J. Wills (éds): Geographies of economics. - London: Arnold: 37-46.

Perkmann, M. (2006): Extraregional linkages and the territorial embeddedness of multinational branch plants. Evidence from the South Tyrol region in Northeast Italy. - In: Economic Geography 82: 421-441.

Postel, N. (2008): Les approches du marché. - In: Alternatives Economiques, Hors-Série 77: 20-25.

Rozenblat, C. \& P. Tissandier (2007): Commuters graphs and cities' polycentric cohesion. - In: INSTITUT DE GÉographie de L'Université de Lausanne (éd.): Proceedings of the $15^{\text {th }}$ European Colloquium on Theoretical and Quantitative Geography. - Lausanne: Faculté des Géosciences et de l'Environnement: 345-355.

SAXENIAN, A. (1994): Regional advantages. Culture and competition in Silicon Valley and Route 128. - Cambrige: Harvard University Press.

Simiand, F. (1912): La méthode positive en science économique. - Paris: Félix Alcan.

Steiner, P. (1999): La sociologie économique. - Paris: La Découverte.

Swedberg, R. (1994): Une histoire de la sociologie économique. - Paris: Desclée de Brouwer.

SwEDBERG, R. (2006): Quand la sociologie économique rencontre l'économie des conventions. - In: EyMARDDunernay, F. (éd.): L'économie des conventions, méthodes et résultats. - Paris: La Découverte: 77-92.

Veblen, T. (1898): Why is economics not an evolutionary science? - In: The Quarterly Journal of Economics 12: 373-397.

Veltz, P. (1996): Mondialisation, villes et territoires. L'économie d'archipel. - Paris: Presses Universitaires de France. 
VidAl de la Blache, P. (1903): Tableau géographique de la France. - Paris: Hachette.

Walliser, B. (2000): L'économie cognitive. - Paris: Odile Jacob.

Williamson, O.E. (1985): The economic institutions of capitalism. - New York: The Free Press.

\section{Résumé: Nouvelle Sociologie Économique et Nouvelle Géographie Économique. Leur impact en analyse territoriale}

La fertilité croisée de la Nouvelle Sociologie Économique et de la Nouvelle Géographie Économique offre à l'analyse territoriale l'opportunité de réfléchir à la capacité endogène de renouvellement des systèmes régionaux en insistant sur l'importance de l'architecture institutionnelle des espaces géographiques. L'orthodoxie, soit la Nouvelle Géographie Économique, ne fait toutefois encore, en analyse territoriale, guère de place à la Nouvelle Sociologie Économique pour laquelle l'encastrement socioculturel est un enjeu analytique majeur. Tout en ne reniant pas l'éclectisme déstabilisant de la Nouvelle Sociologie Économique, il en est appelé, en géographie économique, à un plus grand irrespect des frontières disciplinaires.

Mots-clés: sociologie économique, géographie économique, analyse territoriale, frontière disciplinaire, encastrement

Summary: Influence of New Economic Sociology and New Economic Geography on space analysis

The exchange of ideas between New Economic Sociology and New Economic Geography draws attention to the importance of institutional architecture in geographic space. This emphasis offers space analysis the opportunity to reflect on the ability of endogenic factors to renew regional systems. Regrettably, despite the challenge faced by New Economic Sociology to analyse sociocultural rootedness, there has not been much interaction with New Economic Geography. Although the destabilizing eclecticism of New Economic Sociology is recognized, it is argued here that Economic
Geography could profit from greater interaction with related scientific fields.

Keywords: economic sociology, economic geography, space analysis, scientific field boundaries, rootedness

Zusammenfassung: Neue Wirtschaftssoziologie und Neue Wirtschaftsgeographie: ihr Einfluss auf Raumanalysen

Der Gedankenaustausch zwischen der Neuen Wirtschaftssoziologie und der Neuen Wirtschaftsgeographie eröffnet für die Raumanalyse die Möglichkeit, endogene Fähigkeiten zur Erneuerung von regionalen Systemen zu überdenken, indem die Wichtigkeit der institutionellen Architektur geographischer Räume betont wird. Allerdings räumt die Lehrmeinung der Neuen Wirtschaftsgeographie der Neuen Wirtschaftssoziologie, für welche die soziokulturelle Verwurzelung eine grosse analytische Herausforderung darstellt, nur wenig Platz ein. Der destabilisierende Eklektizismus der Neuen Wirtschaftssoziologie wird nicht abgestritten, doch soll in der Wirtschaftsgeographie ein stärkeres Hinwegsehen über Fachgrenzen angestrebt werden.

Schlüsselwörter: Wirtschaftssoziologie, Wirtschaftsgeographie, Raumanalyse, Fachgrenze, Verwurzelung

Dr. Christophe Mager, Institut de géographie, Université de Lausanne, Quartier Dorigny, Bâtiment Anthropole, CH-1015 Lausanne et Institut de sociologie, Université de Neuchâtel, Faubourg de l'Hôpital 27, CH-2000 Neuchâtel, Suisse.

e-mail: Christophe.Mager@unil.ch

\section{Manuskripteingang/received/manuscrit entré le} 8.6.2008

Annahme zum Druck/accepted for publication/accepté pour l'impression: 17.11.2008 